SHORT REPORT

\title{
Unidirectional whole body turning: a new lateralising sign in complex partial seizures
}

\author{
G Shukla, M Bhatia, M V Padma Srivastava, M Tripathi, A Srivastava, V P Singh, P Saratchandra, \\ A Gupta, S Gaikwad, C S Bal, S Jain
}

Background: The lateralising significance of unidirectional whole body turning in patients with complex partial seizures (CPS) arising from the temporal lobe was evaluated. Methods: A total of 330 patients undergoing long term video-EEG study were included. "Unidirectional whole body turning" was defined as rotation of the trunk, head, and limbs by $>90^{\circ}$ and lasting $>10$ s. EEG correlates, MRI, and SPECT findings were compared and outcome after surgery was noted for patients with follow up data for $>1$ year. Results: Unidirectional whole body turning was observed in 13 patients with a mean age of $18 \pm 8$ years. Concordance of the side of whole body turning with the EEG focus and MRI findings was observed in 11 of the 13 patients $(84.7 \%$ ) and in 26 of 28 seizures (92.8\%). The six patients who underwent temporal lobectomy or resection of lesion, opposite to the direction of body turning, had good seizure outcome.

Conclusion: Unidirectional whole body turning is a new lateralising sign in temporal lobe CPS with good predictive value for epileptogenic focus contralateral to the direction of turning.

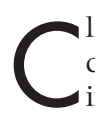
linical behavioural characteristics, especially during complex partial seizures (CPS), have long generated immense interest among neurologists. Due to the increasing numbers of centres for epilepsy surgery, the semiology of CPS has become a valuable tool not only for classification, ${ }^{1}$ but also, more importantly, for lateralisation of epileptogenic foci. Several useful signs such as dystonic limb posturing, ${ }^{2}$ head and eye version, ${ }^{3}{ }^{4}$ ictal speech, and postictal aphasia/dysnomia ${ }^{5}$ have been described for lateralisation and localisation of seizure foci in intractable CPS. However, it is desirable to determine the localising value of all signs occurring in patients with intractable CPS, since none of these signs occur in all patients. We observed an interesting clinical sign of unidirectional turning of the whole body in patients with intractable CPS undergoing evaluation for epilepsy surgery. We have previously described this sign, ${ }^{6}$ however, on observing it in more patients, we sought to evaluate its significance for lateralisation of seizure focus. There are no other reports focussing attention on this phenomenon in CPS.

\section{METHODS}

Videotapes of all patients with intractable CPS undergoing evaluation for epilepsy surgery at the Department of Neurology, All India Institute of Medical Sciences during the period January 1998 to June 2003, were reviewed independently by two neurologists (GS and MB). VideoEEG had been performed for these patients after prior admission and tapering of antiepileptic medication. Ictal EEG was recorded using the 10-20 International system, with application of anterior temporal electrodes in all patients. ${ }^{7}$

Patients with unidirectional whole body turning formed the study group. This sign was defined as rotation of the trunk (with head and limbs) in one direction by more than $90^{\circ}$, lasting for $\geqslant 10 \mathrm{~s}$. Occurrence of head and eye version ${ }^{3}$ and dystonic limb posturing ${ }^{2}$ in addition to turning of the whole body, was noted. During the review of video recordings, authors were blinded to the clinical and investigational details of the patients.

EEG correlates were reviewed after noting the clinical details from videotapes. Precise clinical and EEG correlation was ensured by using a clock generated time signal appearing simultaneously on the video screen and on the digital EEG computer screen. Details of MRI tailored for evaluating intractable epilepsy patients and interictal SPECT were noted for all patients, while findings on ictal SPECT done in some patients, were also noted. Data regarding date and type of surgery and follow up data were reviewed.

Significant seizure reduction $>90 \%$ or seizure freedom for at least 1 year following surgery was considered the gold standard when determining the value of unidirectional whole body turning in lateralisation of seizure foci. For patients in whom adequate follow up data were unavailable, concordance with ictal EEG and MRI findings was determined.

\section{RESULTS}

Long term video-EEG recording was carried out in a total of 330 patients over the study period. Thirteen patients (eight males, five females) with a mean age of $18 \pm 8.2$ years (range 10-38 years) were found to have unidirectional whole body turning during at least one of their recorded seizures (table 1). Patients had experienced seizures for an average of $11.5 \pm 7.3$ years, ranging from 2 to 26 years. Median seizure frequency was 2-3 per month. All patients included were right handed. The total number of seizures in which this sign was observed was 28 , with a median of 2 seizures per patient. Five patients demonstrated turning of the whole body towards the left, seven had turning towards the right, while one patient had turning to the left in one seizure and right in another seizure. In all patients, turning consisted of rotation of the trunk by greater than $90^{\circ}$ towards one side, which in most seizures resulted in the patient almost completing a circle (fig 1).

Head and/or eye version ipsilateral to whole body turning was observed in 10 patients, while ipsilateral dystonic upper limb posturing was seen in six patients; onset of both these phenomena always preceded whole body turning.

Abbreviations: CPS, complex partial seizures; MTS, mesial temporal sclerosis 


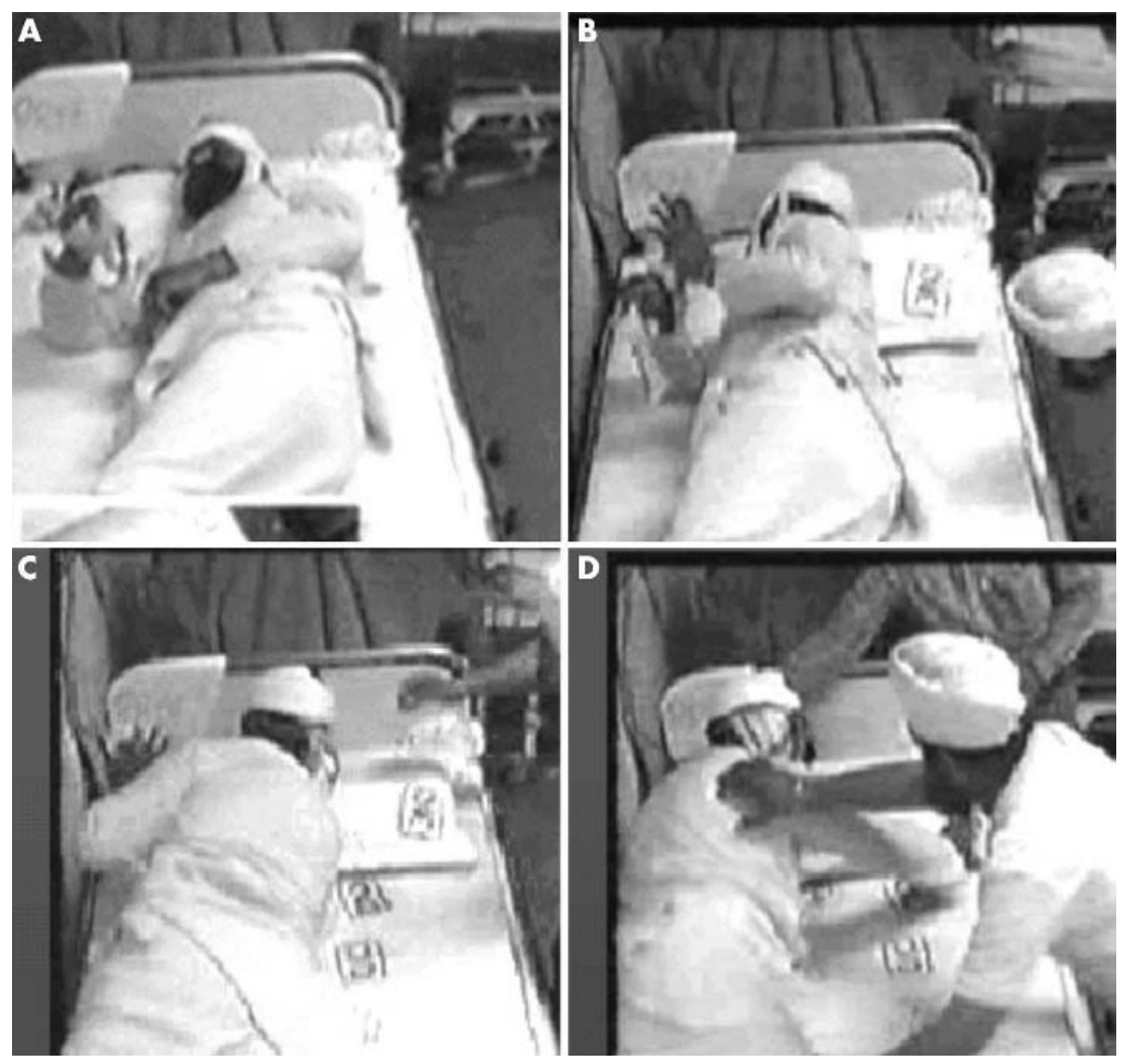

Figure 1 A patient with a left temporal lesion, with EEG focus over the left temporal region, in different stages of whole body turning towards the right during video-EEG recording (the patient shown in this photograph gave consent for its publication).

Lateralising speech abnormalities were not observed in any of these patients. Six patients had secondary generalisation of the seizures with generalised tonic-clonic movements following whole body turning.

Ictal EEG correlates suggested temporal lobe onset in nine patients, while there were hemispheric discharges in four patients. Concordance of side of unidirectional whole body turning with the side of the EEG focus was observed in 11 out of 13 patients $(84.7 \%)$ and in 26 out of 28 seizures $(92.8 \%)$. Interictal EEG in two of these 11 patients showed independent discharges on the side opposite to the seizure focus. In one of these patients, the predominantly involved side (contralateral to whole body turning) was confirmed by ictal SPECT and other concordant findings of ictal EEG and MRI. The other patient had progressive left hemispheric atrophy suggestive of Rasmussen's encephalitis (with intractable temporal lobe CPS during the first video-EEG study, and later developed epilepsia partialis continua); hence, the independent discharges on the opposite side were ignored and the patient underwent left hemispherotomy and became seizure free. One of the two patients in whom EEG findings were not clearly lateralised, had already been operated on for left sided mesial temporal sclerosis (MTS), with unsatisfactory reduction in seizure frequency, and the postoperative video-EEG showed abrupt appearance of bitemporal discharges at ictal onset. Ictal SPECT carried out postoperatively revealed hyperperfusion on the right temporal lobe (contralateral to the side of whole body turning). The other patient had body turning towards the left in one seizure with unclear ictal EEG correlates and towards the right in another seizure with left temporal EEG focus (concordant). His MRI showed bilateral MTS (right more than left), while interictal and ictal
SPECT showed right temporal localisation. This patient was operated on for right temporal lobectomy and was seizure free at 42 months of follow up.

Table 1 Clinical, EEG, and MRI characteristics of the 13 patients (with a total of 28 seizures) who demonstrated unidirectional whole body turning

\begin{tabular}{ll}
\hline Characteristic & Observations \\
\hline Gender ratio (M/F) & $8: 5$ \\
Age (years), mean (range) & $18 \pm 8(10-38)$ \\
Seizure duration (years), mean (range) & $11.5 \pm 7.3$ (2-26) \\
Seizure frequency, median per month & $2-3$ \\
Seizures recorded per patient, median & 2 \\
Direction of turning, no. of patients & 7 \\
Right & 5 \\
Left & 1 \\
Right and left & \\
EEG focus & $11(84.7)$ \\
Contralateral & $26(92.8)$ \\
$\quad$ No. of patients (\%) & 2 (15.3) \\
No. of seizures (\%) & $3(10.7)$ \\
Ipsilateral & \\
$\quad$ No. of patients (\%) & 4 \\
No. of seizures (\%) & 2 \\
MRI findings, no. of patients & 2 \\
Bilateral (asymmetrical) MTS & 1 \\
Unilateral MTS & 1 \\
Temporal lobe cystic lesions & 1 \\
Rasmussen's encephalitis & 2 \\
Medial temporal DNET & \\
Temporal post-traumatic gliosis & \\
Normal MRI & \\
\hline DNET, dysembryoplastic neuroepithelial tumour; MTS, mesial temporal \\
sclerosis.
\end{tabular}


In nine of these 11 patients, MRI and interictal SPECT also showed a lesion and hypoperfusion on the side opposite to the direction of whole body turning; the other two patients had normal MRI with non-lateralising SPECT findings. An interesting observation was that four of the 13 patients had bilateral but asymmetrical MTS on MRI. Two patients had unilateral MTS, one patient had Rasmussen's encephalitis, one had a mesial temporal dysembryoplastic non-epithelial tumour, and one had post-traumatic gliosis in the temporal region. Two patients had cystic lesions. SPECT showed localisation corresponding to MRI findings. Temporal lobe involvement was present in all patients except for two with normal MRI and non-localising SPECT.

Post surgery follow up data were available for six of the 13 patients. Temporal lobectomy for mesial temporal sclerosis was performed in four, while lesionectomy for a temporal dysembryoplastic neuroepithelial tumour and a cystic lesion was performed in one patient each. Five of these six patients were seizure free after surgery after a mean follow up period of 26 months. The other patient had a greater than $90 \%$ reduction in seizure frequency and experienced minor events only at night. Taking seizure outcome post surgery as the gold standard, the positive predictive value of unidirectional whole body turning is $100 \%$.

\section{DISCUSSION}

This study demonstrates that unidirectional whole body turning is a useful sign for lateralisation in CPS of temporal lobe origin, as it was found to be concordant with the EEG focus in 11 of 13 patients (84.7\%) and in 26 of $28(92.8 \%)$ seizures. Also, all six patients who underwent surgery on the side contralateral to the direction of whole body turning had good seizure outcome. This is the second study to describe unilateral turning of whole body in CPS of temporal lobe origin; it was described by the same authors previously in four patients in a study evaluating the lateralising value of dystonic limb posturing and head version in temporal lobe CPS. ${ }^{6}$ In that study, unidirectional whole body turning was found to be predictive of a contralateral EEG focus in all four patients $(100 \%)$. In the present study, we describe this sign in a larger number of patients. Concordance with EEG foci has also been found to be high in the present study. The details of patients in whom relative discordance was observed have been described. Both patients with discordant EEG foci had bilateral foci, which were almost equally responsible for the intractable seizures. In fact, this sign appears to occur commonly in patients with bilateral pathology, since six out of the 13 patients had bilateral lesions or epileptiform discharges and in these, this sign was found to be valuable for lateralisation. However, keeping in view the small number of patients demonstrating this sign, this assumption may be incorrect.

Unidirectional turning of the whole body as a clinical sign in temporal lobe CPS does not occur very frequently, since it was observed in only 13 out of a total of $330(4 \%)$ patients studied. In a study analysing interobserver variability and accuracy of lateralising signs in 38 patients with intractable partial seizures, $45 \%$ patients had head version, 37\% had upper limb dystonic posturing, $21 \%$ had unilateral upper limb automatisms, and $16 \%$ had ictal speech. ${ }^{8}$ However, in that study, only those patients with good post surgery seizure outcome were studied. Hence, it is possible that if all patients being evaluated for epilepsy surgery were included, the frequency of occurrence of these lateralising signs would be much less.

Except for our previous report, there is no other mention of whole body unidirectional turning in studies on the clinical semiology of CPS of either temporal or extratemporal onset. ${ }^{1-59}$ In a recent study by Kotagal et al, ${ }^{10}$ the authors have classified looking around, turning over, sitting up, and walking, as whole body automatisms. However, unidirectional sustained turning was not mentioned. Truncal movements as manifestations of sexual automatisms were initially believed to be features of frontal lobe CPS. ${ }^{11}$ However, it has been proven in studies on patients with such automatisms operated on for temporal lobectomy with good surgical outcome, that the origin of truncal and pelvic movements along with genital automatisms is in the temporal lobe. ${ }^{12}$

Unidirectional whole body turning could be confused with circling which can occur in other seizure types, for example in the primary generalised epilepsies. The sign observed by us was one of several signs seen in temporal lobe CPS. The circling seizures described in the literature describe more of an automatic behaviour in the form of circling rather than unidirectional sustained turning of the whole body. Moreover, even in patients with circling or rotational seizures, onset is possibly from the temporal lobes with spread to the basal ganglia or deeper structures, as shown in the study by Vercueil et al, ${ }^{13}$ which may suggest that these are temporal lobe CPS, irrespective of the syndrome in which they may occur.

We postulate that the occurrence of unilateral whole body turning is due to spread of the epileptiform discharges from the mesial temporal lobe to involve the corticoreticulospinal pathways leading to righting behaviour such as truncal movements in the direction opposite to the temporal lobe predominantly affected. Experimental studies on the lamprey model have demonstrated that the middle rhombencephalic reticular nucleus may have an important role in the generation of turning behaviour. ${ }^{14}$ There is also evidence that, while straight movements in this model are due to alternating bursts of motor activity with equal duration and spike frequency on both sides of the body, for turning, stimulation of crossing inhibitory neurons led to a rise in spike frequency and burst duration on the turning side and suppression of activity on the contralateral side. ${ }^{15}$ To prove this in humans appears difficult. Focussed ictal SPECT studies in all patients demonstrating this sign may be helpful.

Another aspect of body turning in CPS could be the turning bias acquired by humans as a part of normal motor behavioural patterns. It was demonstrated by direct observational study of whole body turning bias in 41 normal adults, that most of the 27 right handed subjects showed significant leftward turning bias, while the 14 left handed individuals did not demonstrate this. ${ }^{16}$ If turning bias was the basis of the direction of turning in temporal lobe CPS, most of our patients (all right handed) would have left sided turning of the whole body, but we observed rightward turning in the majority of patients (seven out of 13). This only confirms that unidirectional body turning is an epileptic phenomenon related to the side of the epileptogenic focus and not just to the handedness of the patient.

The limitation of our study is the small number of patients in whom we observed the new clinical sign of unidirectional whole body turning. However, the total number of patients studied was fairly large. A higher frequency of the occurrence of this sign could increase the reliability of its predictive value in lateralisation of seizure focus.

We conclude by emphasising that unidirectional whole body turning is a reliable, but infrequently occurring, lateralising sign in temporal lobe CPS, not described previously by other authors. Careful observation for this sign can help to lateralise the seizure focus to the side contralateral to the direction of body turning.

\section{Authors' affiliations}

G Shukla, M Bhatia, M V Padma Srivastava, M Tripathi, A Srivastava,

S Jain, Department of Neurology, Comprehensive Epilepsy Care Programme, All India Institute of Medical Sciences, New Delhi, India 
V P Singh, P Saratchandra, A Gupta, Department of Neurosurgery, Comprehensive Epilepsy Care Programme, All India Institute of Medical Sciences, New Delhi, India

S Gaikwad, Department of Neuroradiology, Comprehensive Epilepsy Care Programme, All India Institute of Medical Sciences, New Delhi, India

C S Bal, Department of Nuclear Medicine, Comprehensive Epilepsy Care Programme, All India Institute of Medical Sciences, New Delhi, India

Competing interests: none declared

The patients described in this report gave their informed consent for their details to be published

Correspondence to: Dr Garima Shukla, Department of Neurology, All India Institute of Medical Sciences, New Delhi - 110029, India; garimas@aiims.ac.in

Received 1 April 2004

Revised version received 21 February 2005

Accepted 7 March 2005

\section{REFERENCES}

1 Luders H, Acharya J, Baumgartner C, et al. Semiological seizure classification. Epilepsia 1998;39(9): 1006-13.

2 Kotagal $\mathrm{P}$, Luders $\mathrm{H}$, Morris $\mathrm{HH}$, et al. Dystonic posturing in complex partial seizures of temporal lobe onset: a new lateralizing sign. Neurology 1989;39:196-201.

3 Wyllie $\mathrm{E}$, Luders $\mathrm{H}$, Morris $\mathrm{HH}$, et al. The lateralizing significance of versive head and eye movements during epileptic seizures. Neurology 1986;36:606-11.
4 Marks WJ, Laxer KD. Semiology of temporal lobe seizures: value in lateralizing the seizure focus. Epilepsia 1998;39(7):721-6.

5 Williamson PD, Thadani VM, French JA, et al. Medial temporal lobe epilepsy: videotape analysis of objective clinical seizure characteristics. Epilepsia 1998;39(11):1182-8.

6 Shukla $G$, Bhatia $M$, Gaekwad SB, et al. The lateralizing significance of version of head and dystonic limb posturing in epileptic seizures. Neurology India 2002;50:33-6.

7 Goodin DS, Aminoff MJ, Laxer KD. Detection of epileptiform activity by different noninvasive EEG methods in complex partial epilepsy. Ann Neurol 1990;27(3):330-4.

8 Chee MWL, Kotagal P, Van Ness PC, et al. Lateralizing signs in intractable partial epilepsy: blinded multiple-observer analysis. Neurology 1993:43:2519-25.

9 Olbrich A, Urak L, Groppel G, et al. Semiology of temporal lobe epilepsy in children and adolescents: value in lateralizing the seizure onset zone. Epilepsy Res 2002;48:103-10.

10 Kotagal P, Arunkumar G, Hammel J, et al. Complex partial seizures of frontal lobe onset: statistical analysis of ictal semiology. Seizure 2003;12:268-81

11 Spencer SS, Spencer DD, Williamson PD, et al. Sexual automatisms in complex partial seizures. Neurology 1983;33(5):527-33.

12 Leutmezer F, Serles W, Bacher J, et al. Genital automatisms in complex partial seizures. Neurology 1999;52(6):1188-91.

13 Vercueil L, Kahane $P$, Francois-Joubert A, et al. Basal ganglia involvement in rotational seizures. Epileptic Disord 1999;1(2):107-12.

14 Fagerstedt P, Orlovsky GN, Deliagina TG, et al. Lateral turns in the lamprey. II. Activity of reticulospinal neurons during the generation of fictive turns. J Neurophysiol 2001;86(5):2257-65.

15 Kozlov AK, Ullen F, Fagerstedt P, et al. Mechanisms for lateral turns in lamprey in response to descending unilateral commands: a modeling study. Biol Cybern 2002;86(1):1-14

16 Yazgan MY, Leckman JF, Wexler BE. A direct observational measure of whole body turning bias. Cortex 1996;32(1):173-6. 